\title{
Ritmos de crecimiento diamétrico en los bosques secos tropicales: aportes al manejo sostenible de los bosques de la provincia biogeográfica del Cerrado Boliviano
}

\author{
Diameter growth rates in tropical dry forests: contributions to the sustainable management \\ of forests in the Bolivian Cerrado biogeographical province
}

\author{
Lidio López a,b *, Ricardo Villalba ${ }^{\text {a }}$, Marielos Peña-Claros ${ }^{\mathrm{c}}$

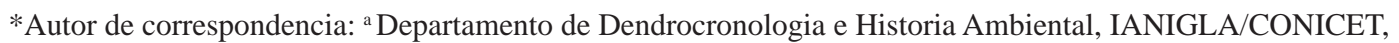 \\ C. Correo 330-5500, Mendoza, Argentina, lopez@mendoza-conicet.gob.ar \\ b Universidad de Valladolid, Instituto Universitario de Investigación, Gestión Forestal Sostenible, \\ ETS de Ingenierías Agrarias, 34004, Palencia, España. \\ c Instituto Boliviano de Insvestigación Forestal, IBIF, 6204, Santa Cruz, Bolivia, \\ Forest Management Group, Wageningen University, P.O. Box 47, 6700 AA Wageningen, Holanda.
}

\begin{abstract}
SUMMARY
Growth ring variations were used to provide the rates in diameter growth for seven tree species in the Bolivian Cerrado biogeographical province. Ten to 50 trees were measured per species. Ring width measurements provided accurate data on the rates of tree growth. Variations in growth rates were determined among species and among sites for the same species over a common period of 100 years. Diametric increases range from 0.43 to $0.56 \mathrm{~cm}_{\text {year }}{ }^{-1}$ in Chiquitano biogeographical district. For species in Guarayos district, diametric increments range from 0.51 to $1.06 \mathrm{~cm}_{\text {year }}^{-1}$. For Centrolobium microchaete growing in both districts, the annual diametric increments oscillate between 0.35 to $0.40 \mathrm{~cm}_{\text {year }}{ }^{-1}$ and 0.55 to $0.65 \mathrm{~cm}$ year ${ }^{-1}$ in Chiquitano and Guarayos districts, respectively. Diametric increases in Chiquitano district were not significantly different among sites, whereas in Guarayos district, annual increases differ among species and from the same species growing in Chiquitano district. Observed diameter growth rates indicate that diametrical increments are generally slower than commonly assumed, with considerable variation among species and between districts. Therefore, the idea of applying similar rules for the management of different species across different sites without considering actual growth rates is not recommended, with long-term consequences for the sustainability of forests at the tropical dry Bolivian Cerrado.
\end{abstract}

Key words: rings of growth, diameter growth rates, current annual increment, and forest management.

\section{RESUMEN}

La información obtenida a partir de los anillos de crecimiento fue utilizada para proveer los ritmos de crecimiento diamétrico de siete especies forestales de la provincia biogeográfica del Cerrado Boliviano. En este análisis dendrocronológico se emplearon 10 a 50 árboles por especies. La medición del ancho de los anillos de crecimiento brindó datos precisos, con los cuales se determinó la variabilidad del incremento entre especies y entre sitios para un periodo común de 100 años. Los incrementos diamétricos variaron de 0,43 a 0,56 cm año-1 en especies correspondientes al distrito Chiquitano. Para especies del distrito de Guarayos, los valores fueron de 0,51 a 1,06 cm año ${ }^{-1}$. Para Centrolobium microchaete que crece en ambos distritos, los valores medios de crecimiento por rodal oscilaron entre 0,35 y 0,40 cm año $0^{-1}$ en el distrito Chiquitano y de 0,55 a 0,65 cm año ${ }^{-1}$ en el de Guarayos. Los incrementos diamétricos en sitios del distrito Chiquitano no presentaron diferencias significativas, en cambio en Guarayos los incrementos fueron diferentes entre especies y, a su vez, diferentes a los del distrito Chiquitano. Las tasas de crecimiento diamétrico indican que el crecimiento diamétrico, en general, es más lento de lo comúnmente asumido, con variaciones importantes entre especies y entre sitios de crecimiento. Por ello, la idea de establecer pautas fijas de manejo similares para todas las especies y sitios, sin considerar las tasas reales de crecimiento, no sería la recomendada, poniendo en algunos casos en riesgo la sostenibilidad de los bosques "manejados" del cerrado boliviano.

Palabras clave: anillos de crecimiento, tasas de crecimiento diamétrico, incremento corriente anual, manejo forestal.

\section{INTRODUCCIÓN}

Los bosques tropicales y subtropicales de América del Sur presentan una alta diversidad de especies maderables que proveen significantes volúmenes de madera a los mercados domésticos e internacionales (Kant 2004). Solo en
Bolivia existen más de 150 especies arbóreas maderables de las cuales 13 a 15 especies son actualmente aprovechadas (BOLFOR II 2009, Mostacedo et al. 2009). La superficie de bosques destinados a la producción forestal es de 28 millones de hectáreas de las cuales nueve millones están siendo actualmente aprovechadas. Un 52 \% de estas áreas 
aprovechadas se encuentran en los bosques del Cerrado Boliviano (BOLFOR II 2008). Estos bosques se componen de ecosistemas muy diversos siendo, por ello, los más ricos en especies forestales y los más extensos de Bolivia (Killeen et al. 1993, Mostacedo et al. 2009)

En Bolivia, la actividad forestal está regulada por tres pautas principales de manejo: un ciclo de corte de 20 años, una tasa máxima de extracción del $80 \%$ y la fijación del límite diamétrico de corte que varía entre 40 a $70 \mathrm{~cm}$ dependiendo de la especies. Existen otros requisitos obligatorios a cumplir previamente a la explotación forestal tendientes a lograr el rendimiento sostenible del bosque incluyendo la evaluación del efecto del aprovechamiento mediante el monitoreo en parcelas permanentes de muestreo en el bosque (MDSMA 1997). Sin embargo, estas pautas forestales fueron determinadas sobre bases teóricas y elaboradas con datos de crecimiento procedentes de otros bosques tropicales, que no reflejan precisamente la realidad productiva de los bosques bolivianos (Dauber et al. 2005, López 2010). Ante la falta de información, estas directrices de gestión forestal fueron una alternativa provisoria al manejo forestal. Sin embargo, hasta el día hoy no han sido contrastadas con datos de campo, al menos para las especies más explotadas comercialmente (López 2010). En Bolivia existe una Red Nacional de Parcelas Permanentes de muestreo situadas en las principales eco-regiones, cuyas mediciones se iniciaron en el año 1996 y que, hasta la actualidad, han provisto considerable información para el manejo forestal (Dauber et al. 2005). Si bien estos datos dan una idea sobre la ecología y los ritmos de crecimiento para algunas especies, la corta extensión temporal de estos registros no ha permitido evaluar los impactos y validar biológicamente las pautas forestales propuestas inicialmente.

En algunas regiones tropicales, los anillos de crecimiento han sido empleados exitosamente para determinar la edad de los árboles tropicales (Brienen y Zuidema 2006a). La medición del anchos de los anillos de crecimiento, también ha sido empleada para cuantificar en forma precisa las tasas de crecimiento de una masa forestal (Brienen y Zuide- ma 2006a). Asimismo, la información obtenida a partir de los anillos de crecimiento ha permitido establecer los turnos de corte y los ciclos de corte para pocas especies tropicales (Brienen y Zuidema 2006b, Schöngart 2008). Los anillos de crecimiento de algunas especies de los bosques tropicales han sido incluso empleados para establecer las relaciones entre las variaciones en el clima regional y el crecimiento radial (Brienen y Zuidema 2005, López y Villalba 2010).

Dado que en Bolivia, las pautas forestales se basan en turnos fijos de rotación que no tienen en cuenta los ritmos de crecimiento de cada especie en diferentes tipos de bosque, la hipótesis de trabajo es que la determinación de estos ritmos de crecimiento proveería información muy valiosa para establecer turnos forestales destinados al manejo sostenible de los bosques tropicales. El objetivo específico de este trabajo es proveer las tasas de crecimiento diamétrico, a partir de la medición de los anillos de crecimiento, de siete especies maderables del Cerrado Boliviano. En este trabajo, las tasas de crecimiento diamétrico de siete especies maderables del Cerrado Boliviano, provista por los anillos de crecimiento, se emplean para determinar la variabilidad del crecimiento diamétrico entre especies y entre sitios con condiciones ambientales diferentes para una misma especie. Por ello, los registros dendrocronológicos proveerán bases sólidas para dar recomendaciones estratégicas en el manejo sostenible del bosque boliviano.

\section{MÉTODOS}

Área de estudio. Los sitios de estudio están localizados en dos distritos de la provincia biogeográfica del Cerrado Boliviano (Navarro y Maldonado 2004). La provincia del Cerrado es una de las más extensas de América del Sur, extendiéndose desde el centro-norte de Brasil hasta el este de Bolivia y noreste de Paraguay (Cabrera y Willnk 1973). La provincia biogeográfica del Cerrado Boliviano está compuesta por cinco distritos biogeográficos (Navarro y Maldonado 2004). Los sitios de muestreo se encuentran en los distritos de Guarayos y Chiquitano (cuadro 1).

Cuadro 1. Localización geográfica de los sitios de estudio dentro de la provincia biogeográfica del Cerrado Boliviano. Las letras indican la situación del bosque de (PP) propiedad privada, (TCO) tierras comunitarias de origen y (CF) concesiones forestales.

Geographical location of the study sites within the biogeographical province of Bolivian Cerrado. The letters indicate the status of forest as follows: (PP) private property, (TCO) community lands, and (CF) forest concessions.

\begin{tabular}{|c|c|c|c|c|c|}
\hline Región & Nombre del sitio & Situación del bosque & $\begin{array}{c}\text { Latitud } \\
\text { S }\end{array}$ & $\begin{array}{c}\text { Longitud } \\
\mathrm{O}\end{array}$ & $\begin{array}{c}\text { Altitud } \\
\text { (m s.n.m.) }\end{array}$ \\
\hline \multirow[t]{3}{*}{ Distrito Chiquitano } & Inpa & PP & $16^{\circ} 22^{\prime}$ & $61^{\circ} 55^{\prime}$ & 503 \\
\hline & Santa Anita & TCO & $16^{\circ} 32^{\prime}$ & $61^{\circ} 55^{\prime}$ & 423 \\
\hline & Zapocó & TCO & $16^{\circ} 27^{\prime}$ & $61^{\circ} 44^{\prime}$ & 464 \\
\hline \multirow[t]{3}{*}{ Distrito de Guarayos } & La Chonta & $\mathrm{CF}$ & $15^{\circ} 38^{\prime}$ & $62^{\circ} 46^{\prime}$ & 250 \\
\hline & Santa Mónica & TCO & $15^{\circ} 58^{\prime}$ & $62^{\circ} 22^{\prime}$ & 420 \\
\hline & Makanaté & TCO & $15^{\circ} 29^{\prime}$ & $62^{\circ} 17^{\prime}$ & 246 \\
\hline
\end{tabular}


Estos distritos incluyen bosques que se extienden entre las formaciones húmedas tropicales y sus aéreas de transición (Chiquitano-Amazónico) hasta las sabanas húmedas del pantanal y los bosques caducifolios del Chaco boliviano (Killeen et al. 1993).

Estos dos distritos cubren una gran amplitud territorial y cuentan con una alta biodiversidad característica de ambientes tropicales estacionales. Particularmente los bosques de estos distritos están dominados por leguminosas (Killeen et al. 1993). El dosel superior alcanza alturas mayores en los Bosques de Guarayos (20 a 30 m) que en el Chiquitano (15 a $25 \mathrm{~m}$ ).

El distrito biogeográfico Chiquitano presenta, en general, suelos poco profundos y muy pedregosos sobre relieves dominados por serranías y mesetas. Por su parte, el distrito de Guarayos tiene suelos moderadamente profundos a muy profundos, con mayor capacidad de retención de humedad (Navarro y Maldonado 2004). Las temperaturas medias de las estaciones meteorológicas de Concepción en Chiquitos y Ascensión de Guarayos, ubicadas en ambos distritos son de $24,2^{\circ} \mathrm{C}$ (intervalo $1943-2006$ ) y $23,8^{\circ} \mathrm{C}$ (intervalo 1947-2006), respectivamente. La precipitación total anual, caracterizada por una marcada estacionalidad, varía entre 1.090 y $1.270 \mathrm{~mm}$ en Concepción (periodo 1943-2010) y 1.400 a 1.600 mm en Guarayos (periodo 1943-2010). Dados los menores niveles de precipitación en Concepción, la estación seca invernal tiene una duración media de 6 meses (abril-septiembre), un mes más que en Guarayos (mayo-septiembre). Las diferencias en la cantidad total de precipitación y la duración de la estación seca determinan que los bosques del distrito biogeográfico de Guarayos tengan más elementos característicos de los bosques tropicales húmedos que los bosques Chiquitanos.

Los seis sitios se encuentran dentro de las regiones forestales productoras de maderas comercializadas ac- tualmente en Bolivia (BOLFOR II 2008). Todos los sitios cuentan con bosques manejados, uno de ellos pertenece a la categoría de concesión forestal (Guarayos-La Chonta) con una superficie de bosque de 100 mil hectáreas y la propiedad privada (INPA) con 30 mil hectáreas, respectivamente (BOLFOR II 2009). Mientras que los sitios Santa Mónica, Santa Anita, Zapocó y Makanaté corresponden a bosques manejados por comunidades originarias (TCO), cuyas superficies individualmente no superan las $10 \mathrm{mil}$ hectáreas (cuadro 1). Los sitios de La Chonta, Concepción y Santa Mónica, tienen el total de sus áreas certificadas por la Forest Stewardship Council (FSC) (BOLFOR II 2008). El manejo forestal correspondiente al distrito biogeográfico de Guarayos, está regulado por un diámetro mínimo de corte (diámetro medio cuadrático, DMC) a árboles con diámetros igual o superior $50 \mathrm{~cm}$. Por su parte, en los sitios del distrito Chiquitano, el DMC es de $40 \mathrm{~cm}$. En ambos distritos, el ciclo de corte (rotación entre cosechas) esta propuesto a 25 años, una intensidad del 80 \% y preservando el 20 \% árboles aprovechables para semilleros (BOLFOR II 2008).

Especies en estudio. La selección de las siete especies forestales se basó en la determinación previa de la visibilidad de sus anillos de crecimiento (López 2003). Estas especies forestales son intensamente explotadas para obtener maderas, por lo que su uso actual determinó la obtención de muestras dendrocronológicas suficientes para cada especie (cuadro 2). Estas especies se diferencian en la estructura adulta, las copas de los árboles se encuentran en la capa superior del dosel del bosque y, teóricamente, tienen un alto potencial de crecimiento. Las especies Amburana cearensis (Allemão) A. S. Smith, Cedrela fissilis Vell., Platimiscium ulei Harms y Centrolobium microchaete (C. Martius ex Benth.) Lima ex G. P. Lewis tienen las maderas más

Cuadro 2. Tasas de incremento diamétrico anual, máximo, promedio y mínimo por especies y distrito biogeográfico. Número de ejemplares incluidos por especies (periodo de comparación $=100$ años).

Annual diameter increment rates, maximum, mean and minimum per species and biogeographical district. Number of specimens for species is also indicated (period for comparison $=100$ years).

\begin{tabular}{lcccc}
\hline \multicolumn{1}{c}{ Especies en estudios } & & $\begin{array}{c}\text { Incremento medio anual } \\
\text { (cm año-1 }\end{array}$ & \multicolumn{2}{c}{$\begin{array}{c}\text { Número de árboles } \\
\text { de la muestra }\end{array}$} \\
\hline Nombre científico & Máximo & Promedio & Mínimo & 35 \\
\hline Amburana cearensis & 1,93 & 0,56 & 0,22 & 31 \\
Anadenanthera colubrina & 1,98 & 0,45 & 0,20 & 30 \\
Platimiscium ulei & 1,71 & 0,43 & 0,27 & 31 \\
$\quad$ Promedio regional (D. Chiquitano) & 1,87 & 0,48 & 0,23 & 13 \\
Ficus boliviana & 3,43 & 1,06 & 0,47 & 36 \\
Hymenaea courbaril & 3,01 & 0,57 & 0,36 & 27 \\
Cedrela fissilis & 2,91 & 0,51 & 0,13 & 25 \\
\hline
\end{tabular}


finas y valiosas del Cerrado Boliviano (ITTO 1996). Por su parte, Hymenaea courbaril L. y Anadenanthera colubrina (Vell. Conc.) Benth son las especies con maderas más duras de la región y Ficus boliviana C. C. Berg es considerada como la especie de madera menos densa (liviana).

Las siete especies en estudio tienen una distribución exclusivamente tropical y subtropical (Killeen et al. 1993). Las especies Ficus boliviana y Centrolobium microchaete son endémicas y, concretamente, se encuentran en los bosques de la provincia biogeográfica del cerrado boliviano (Killeen et al. 1993). En cambio, Amburana cearensis y Anadenanthera colubrina tienen una amplia distribución en Bolivia y se encuentran también en países limítrofes como Brasil, Perú y el norte de Argentina. Por su parte, Hymenaea courbaril, Platimiscium ulei y Cedrela fissilis se localizan en muchas regiones de Bolivia y tienen una amplia distribución en América, encontrándose desde México, hasta Paraguay y al norte de Argentina (Killeen et al. 1993).

Colección de muestras. En las áreas anuales de aprovechamiento forestal de estos seis sitios, se colectaron secciones transversales del fuste de los árboles en el momento de su abatimiento para obtener maderas. En general, estos árboles presentaban buenas características morfológicas tanto del fuste como la posición de copa. Se eligieron aquellos árboles más característicos de cada sitio y apeados, en lo posible, lo más cercano a la superficie del suelo. El número de muestras por especie fue establecido de acuerdo a la intensidad del aprovechamiento en cada sitio. Las muestras leñosas se obtuvieron mediante el uso de motosierra. Estudios preliminares en las regiones tropicales secas o semisecas de América del Sur indican la dificultad de obtener muestras leñosas empleando métodos dendrocronológicas tradicionales (López 2003). Dada la dureza que caracteriza a la mayoría de las maderas de esta región, no pueden emplearse los barrenos de incremento tradicionales, los que han sido diseñados para maderas de menor densidad. Asimismo, la mayor dificultad en la tarea de delimitar con precisión los anillos de crecimiento en estas especies, obliga a trabajar con secciones transversales del fuste de los árboles, en lugar de los tradicionales núcleos o tarugos de $5 \mathrm{~mm}$ de espesor. Contar con secciones transversales o cuñas de los árboles permite tener una mejor visión del plan leñoso y aumenta la precisión en la determinación y medición del espesor de los anillos de crecimiento. La mayoría de las muestras fueron tomadas a alturas en el fuste que oscilaron entre 1,5 a $2 \mathrm{~m}$, dependiendo del alcance de los contrafuertes en el fuste y de los procesos de apeado y sanidad de los troncos.

Procesamiento de muestras. Las muestras fueron pulidas y posteriormente fechadas visualmente, siguiendo los criterios ya establecidos en dendrocronología (Stokes y Smiley 1968). En las muestras pulidas, a simple vista o con ayuda de una lupa, se identificó el patrón anatómico que facilita la delimitación de los anillos de crecimiento (López 2010). Una vez definido el patrón anatómico que delimita a los anillos de crecimiento, se procedió al fechado y medición del espesor de los anillos de crecimiento. Los anillos anuales fueron asignados al año en el cual comenzó la formación del leño siguiendo la convención para el Hemisferio Sur (Schulman 1956).

En aquellos árboles que presentaron dificultades para realizar una precisa delimitación de los anillos de crecimiento, se fecharon dos o más radios opuestos en la sección transversal de un mismo árbol. Si el número de anillos determinado en un radio no coincidió con el número en el radio opuesto, los radios fueron re-examinados hasta lograr una coincidencia entre ambos. Una vez que se realizó el fechado, se procedió a medir el espesor de los anillos de crecimiento. Los anchos de anillos fueron medidos en una tableta Velmex UniSlide conectada a un contador digital Metronics Quick-Chek QC-10V con una precisión de $0,001 \mathrm{~mm}$. Con base en los valores obtenidos a partir de la medición del ancho de los anillos de crecimiento, se procedió a la construcción de las curvas de crecimiento diamétrico empleando el programa AGE. Este programa, que forma parte de la Librería de Programas para Dendrocronología (DPL, Holmes 1983), estima el incremento diamétrico corriente anual (ICA) y el incremento medio anual (IMA) para cada serie en particular y provee además el promedio de todas los árboles considerados (Holmes 1983). Con el propósito de reducir la variabilidad interanual en las estimaciones del IMA, los valores interanuales fueron suavizados mediante el uso de un filtro digital spline de 15 años (Cook y Peters 1981).

El ICA [1] corresponde al incremento diamétrico del árbol para un año determinado $(t)$.

$\mathrm{ICA}=C A_{t}-C A_{t-1}$

Donde, el ICA se calcula restando el crecimiento acumulado correspondiente al año $t$ menos el correspondiente al año anterior $t-1$, como se observa en la ecuación y $\mathrm{CA}_{t}$ es crecimiento acumulado en el año $t$ [1].

Los incrementos diamétricos corrientes y medios resultan de promediar los valores radiales correspondientes a dos radios (por lo general opuestos) dentro de una misma sección transversal.

$\mathrm{IMA}=\frac{C A_{t}}{\mathrm{t}}$

Donde, el IMA [2] resulta de dividir el crecimiento acumulado (CA) para el año $t$ por $t$ años.

Los incrementos diamétricos corrientes y medios resultaron de promediar los incrementos correspondientes a 
dos radios (por lo general opuestos) dentro de una misma sección transversal. Para ello e independiente de la fecha calendario, se asignó al anillo central (en contacto con la médula) anillo 1 , siendo en cada sección transversal el año $t=1$. A partir de los incrementos diamétricos por especie y por sitio se determinaron las tendencias biológicas del crecimiento y su variabilidad temporal. Esta información es útil también para establecer los periodos de crecimiento óptimos para cada especie en particular (Brienen y Zuidema 2006a). Finalmente, se compararon los incrementos anuales por especies y por sitios correspondientes a un mismo distrito biogeográfico, entre distritos y con datos obtenidos mediante otros métodos (parcelas permanentes). A su vez, las diferencias en el crecimiento entre árboles de Centrolobium microchaete creciendo en los seis sitios diferentes se determinó mediante una prueba estadística de Kruskal Wallis (Di Rienzo et al. 2009). Diferencias estadísticamente significativas $(P<0,05)$ en el incremento diamétrico medio entre localidades fueron evaluadas asumiendo que esta prueba no requiere distribución normal de las variables. Las diferencias de crecimiento fueron evaluadas durante periodos comunes entre sitios, tratando de incluir en los mismos el número de individuos más elevado posible $(\geq 10)$ con base en el número total de individuos colectados.

\section{RESULTADOS}

Diferencias entre especies. En la mayoría de las especies se observó una marcada variación de sus crecimientos diamétricos en el tiempo, siendo inicialmente mayor y más reducido a medida que los árboles alcanzan diámetros más grandes y edades más longevas (figura 1). Las siguientes comparaciones están basadas en un período común de 100 años que resultaron del promedio de un número de individuos por especie y por sitio que osciló entre 13 y 50 árboles (cuadro 2). Los incrementos diamétricos en especies correspondientes al distrito biogeográfico Chiquitano como Amburana cearensis oscilaron entre 0,22 cm y 1,93 $\mathrm{cm}$ año ${ }^{-1}$, dependiendo de la edad de los ejemplares. El incremento promedio de $A$. cearensis en la localidad de Santa Anita fue de $0,56 \mathrm{~cm}$ año-1 (cuadro 2). Por su parte, en la localidad de Concepción, el incremento diamétrico de Anadenanthera colubrina varió entre 0,20 cm y 1,98 $\mathrm{cm}$ año $0^{-1}$, con valores medios de $0,45 \mathrm{~cm}$ año-1, sobre un periodo de 100 años. Las variaciones registradas para Platimiscium ulei en la localidad de Santa Anita fueron desde 0,27 a $1,71 \mathrm{~cm}_{\text {año }}{ }^{-1}$, alcanzando un incremento diamétrico promedio de $0,43 \mathrm{~cm}_{\text {año-1 }}{ }^{-1}$ (cuadro 2).

Las especies correspondientes al distrito biogeográfico de Guarayos también presentan una alta variabilidad en el
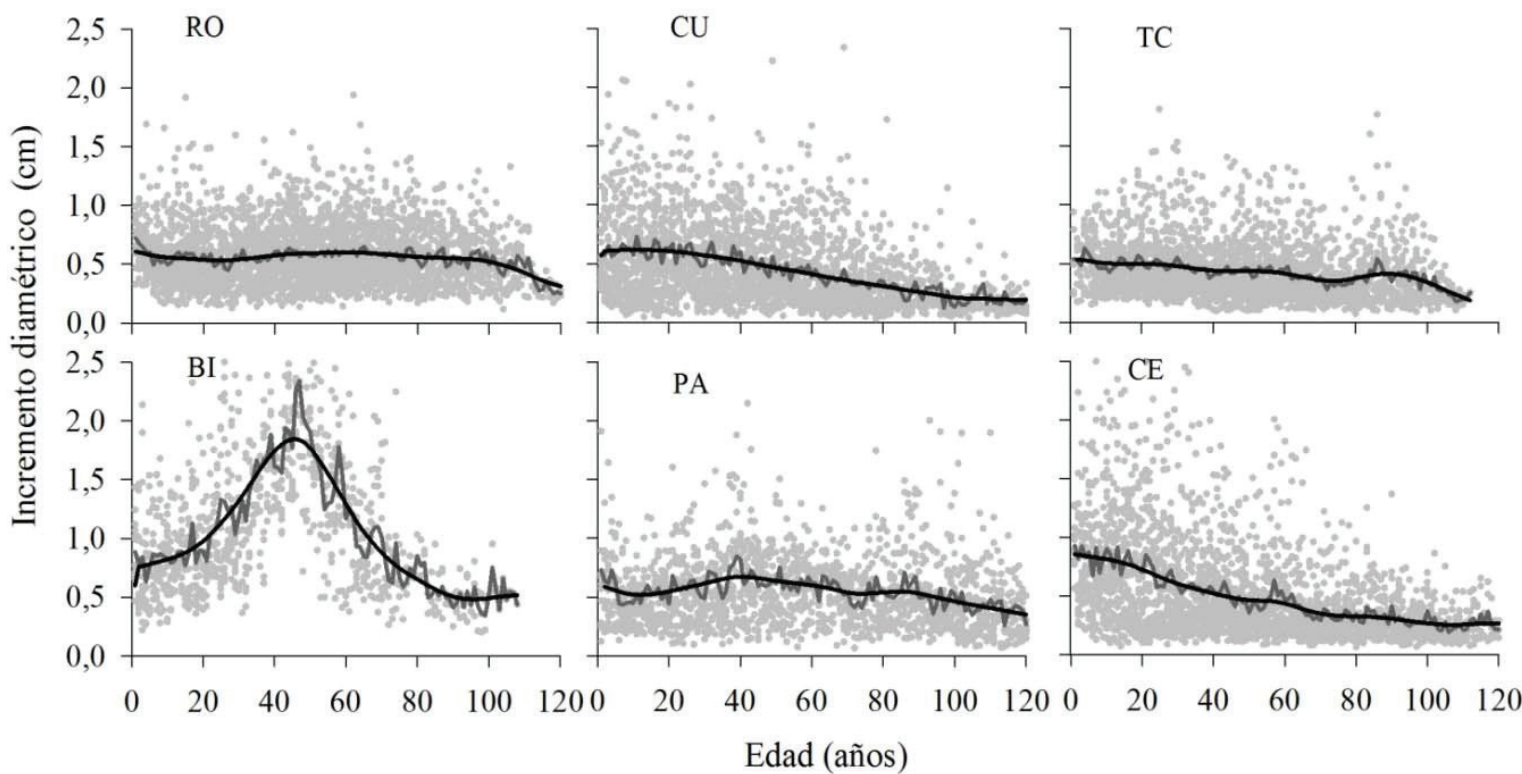

Figura 1. Variaciones del crecimiento diamétrico a través del tiempo para seis especies forestales de la provincia biogeográfica del Cerrado Boliviano. La línea con mayor variación corresponde al incremento medio real y la menos variable (color negro) al suavizado mediante una función spline de 15 años. Las especies (RO) Amburana cearensis, (CU) Anadenanthera colubrina y (TC) Platimiscium ulei, corresponden al distrito Chiquitano y (BI) Ficus boliviana, (PA) Hymenaea courbaril y (CE) Cedrela fissilis, al distrito de Guarayos.

Changes in diametric growth over time for six tree species of the Bolivian Cerrado biogeographical province. The line showing larger interannual variations corresponds to actual mean increment, whereas the smoothed line was obtained using a spline filter of 15 years. The species (RO) Amburana cearensis (CU), Anadenanthera colubrina (CT) and Platimiscium ulei are from Chiquitano district, whereas (BI) Ficus Boliviana (PA), Hymenaea courbaril (EC) and Cedrela fissilis are from Guarayos district. 
crecimiento diamétricos durante los 100 años (figura 1). Especies como Ficus boliviana alcanzaron incrementos diamétricos máximos de hasta $3,43 \mathrm{~cm}$ año-1, en cambio, la misma especie después de los 80 años de edad alcanzó crecimientos cercanos a los $0,46 \mathrm{~cm}$ año-1. En la localidad de Guarayos, los valores de los incrementos diamétricos medios para $F$. boliviana fueron de $1,06 \mathrm{~cm}$ año-1 (cuadro 2). Por su parte Hymenaea courbaril en Guarayos alcanzó tasas de hasta $3,01 \mathrm{~cm}$ año-1, particularmente cuando los árboles eran jóvenes. A medida que los árboles van aumentando en edad y en diámetro, las tasas de crecimiento se redujeron mostrando valores mínimos de hasta $0,36 \mathrm{~cm}_{\text {año }}{ }^{-1}$. Sin embargo, el promedio de $H$. courbaril en La Chonta fue de $0,57 \mathrm{~cm}$ año ${ }^{-1}$, para un intervalo de 100 años de observación. Cedrela fissilis inicialmente tuvo un incremento diamétrico de hasta 2,91 cm año ${ }^{-1}$ durante los primeros 40 años; luego, presentó una marcada reducción hasta alcanzar valores próximos a $0,13 \mathrm{~cm}_{\text {año }}{ }^{-1}$. En síntesis, $C$. fissilis en la localidad de Santa Mónica alcanzó un incremento diamétrico promedio de $0,51 \mathrm{~cm}$ año $0^{-1}$ (cuadro 2).

En general, el promedio regional del incremento diamétrico para las tres especies del distrito Chiquitano fue de $0,43 \mathrm{~cm}$ año ${ }^{-1}$, basado en un promedio de 31 árboles por especie y un periodo común de 100 años de comparación. Por su parte el promedio regional para especies de distrito de Guarayos fue de $0,71 \mathrm{~cm}$ año-1 ${ }^{-1}$ (cuadro 2).
Variación de una especie en sitios diferentes. El material leñoso de Centrolobium microchaete fue colectado en seis sitios, tres en cada uno de los distritos biogeográficos. Con excepción de la localidad de La Chonta, los otros cinco sitios presentaron similares tendencia en los incrementos diamétricos, siendo el crecimiento substancialmente mayor durante los primeros años de crecimiento (figura 2). En cuanto a los valores medios, se observó escasas diferencias entre sitios de un mismo distrito. Los sitios con menor variación correspondieron al distrito Chiquitano, cuyos valores medios oscilaron entre $0,35 \mathrm{~cm}$ año ${ }^{-1}$ en Concepción y 0,40 cm año ${ }^{-1}$ en Santa Anita. En cambio, los sitios del distrito de Guarayos mostraron una mayor variación, los valores medios más bajos fueron de $0,55 \mathrm{~cm}^{2}$ año ${ }^{-1} \mathrm{en} \mathrm{Santa}$ Mónica y los más altos de $0,65 \mathrm{~cm}$ año ${ }^{-1}$ correspondieron a La Chonta (cuadro 3).

A pesar de la escasa diferencia en los crecimientos diamétricos entre sitios de un mismo distrito, la prueba estadística de Kruskal Wallis muestra diferencias significativas entre sitios del distrito de Guarayos. Por ejemplo, de acuerdo a estadístico de Kruskal Wallis, no hubo diferencias significativas para el crecimiento de C. microchaete en los sitios del distrito Chiquitano (cuadro 3). En cambio, el crecimiento en estos sitios fue diferente a los del distrito biogeográfico de Guarayos, en el que a su vez, los sitios de Santa Mónica y La Chonta que muestran niveles de cre-

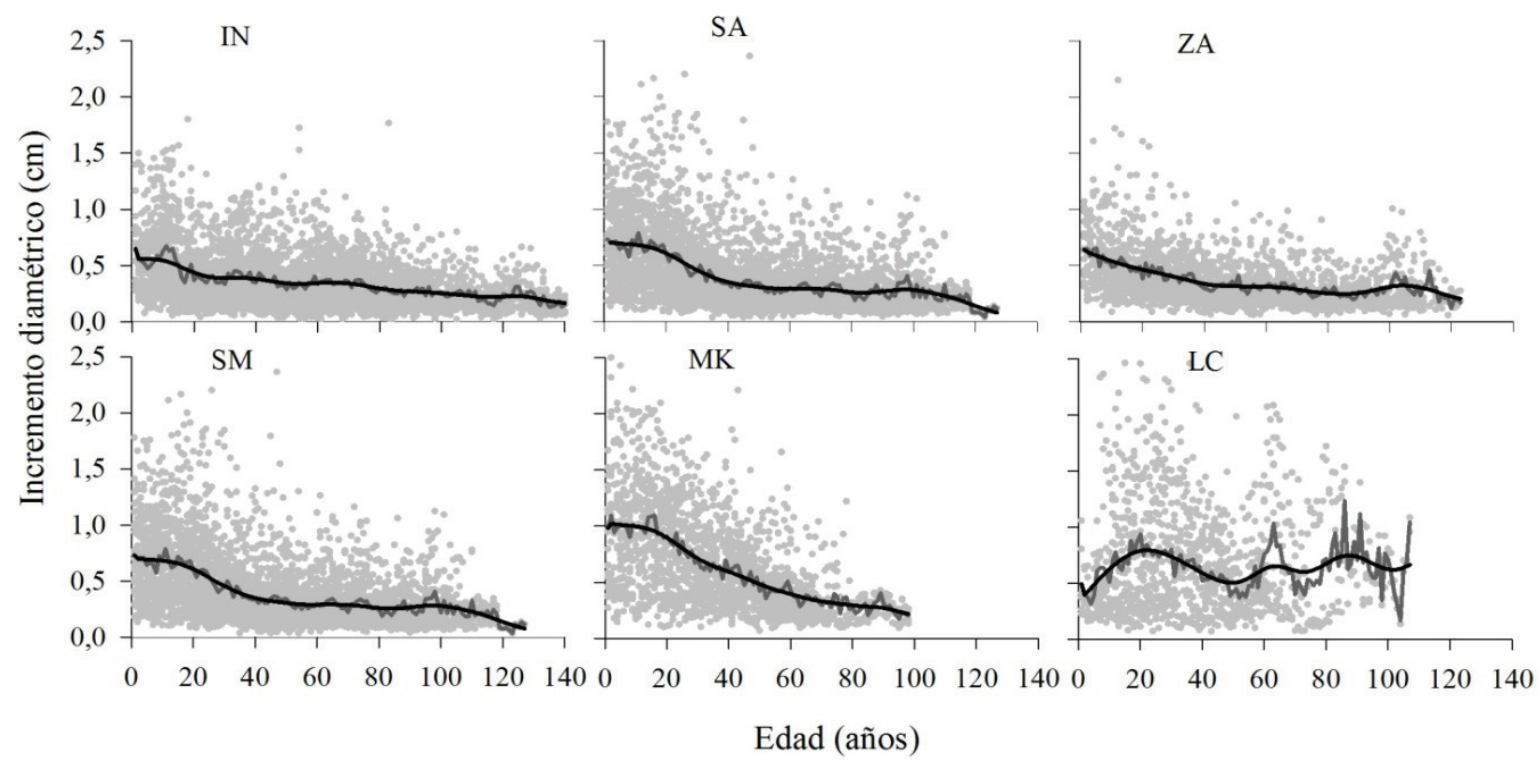

Figura 2. Variaciones del crecimiento diamétrico de Centrolobium microchaete en los seis sitios de estudio. La línea con mayor variación corresponde al incremento medio anual observado y la menos variable al suavizado mediante una función spline de 15 años. Al distrito Chiquitano pertenecen las localidades de (IN) Inpa, (SA), Santa Anita, (ZA), Zapocó y al distrito de Guarayos las localidades de (SM) Santa Mónica, (MK) Makanaté y (LC) La Chonta.

Changes in diametric growth of Centrolobium microchaete in our six study sites. The line showing larger interannual variations corresponds to actual mean increment, whereas the smoothed line was obtained using a spline filter of 15 years. The localities of (IN) Inpa (SA), Santa Anita and (ZA) Zapocó are located in Chiquitano, whereas the localities of (SM) Santa Monica, (MK) Makanaté and (LC) La Chonta in Guarayos biogeographical district. 
Cuadro 3. Prueba de varianza de Kruskal Wallis para estimar diferencias, dentro de un periodo común de comparación de 100 años entre los incrementos medios anuales para Centrolobium microchaete en los seis sitios de muestreo en la provincia biogeográfica del Cerrado Boliviano. Para el incremento medio anual (IMA en $\mathrm{cm}$ ), las diferentes letras (abc) indican diferencias estadísticamente significativas $(P \leq 0,05)$.

Variance test of Kruskal Wallis to determine differences in mean annual diametric increments, estimated over a common period, among six stands of Centrolobium microchaete in the Bolivian Cerrado biogeographic province. For the annual mean increment (IMA in cm), different letters (abc) indicate statistically significant differences among sites $(P \leq 0.05)$.

\begin{tabular}{lccccc}
\hline \multicolumn{1}{c}{ Sitio } & Número de árboles & $\begin{array}{c}\text { IMA } \\
(\mathrm{cm})\end{array}$ & $\begin{array}{c}\text { Promedio del IMA } \\
(\mathrm{cm})\end{array}$ & $\begin{array}{c}\text { IMA máximo } \\
(\mathrm{cm})\end{array}$ & $\begin{array}{c}\text { IMA mínimo } \\
(\mathrm{cm})\end{array}$ \\
\hline Inpa & 38 & $0,37 \pm 0,10 \mathrm{a}$ & 0,37 & 1,80 & 0,09 \\
Santa Anita & 41 & $0,40 \pm 0,46 \mathrm{a}$ & 0,39 & 2,03 & 0,10 \\
Zapocó & 22 & $0,35 \pm 0,11 \mathrm{a}$ & 0,35 & 1,84 & 0,12 \\
Santa Mónica & 50 & $0,55 \pm 0,15 \mathrm{~b}$ & 0,54 & 2,60 & 0,11 \\
La Chonta & 24 & $0,65 \pm 0,17 \mathrm{~b}$ & 0,64 & 2,49 & 0,21 \\
Makanaté & 26 & $0,56 \pm 0,28 \mathrm{c}$ & 0,56 & 2,50 & 0,18 \\
\hline
\end{tabular}

cimiento similares, resultaron ser diferentes de Makanaté (cuadro 3).

\section{DISCUSIÓN}

El empleo de las técnicas dendrocronológicas en especies tropicales es todo un desafío científico, ya que muchas de las especies tropicales presentan dificultades al momento del fechado anual de los anillos de crecimiento (López 2010). Sin embargo, la medición del ancho de los anillos en dos o más radios por árbol, se convierte en una alternativa eficiente y rápida para obtener información aplicable al manejo forestal. Los resultados de este estudio demuestran que los crecimientos diamétricos promedios de las siete especies forestales seleccionadas, son altamente consistentes a los obtenidos mediante la medición de árboles por más de 10 años en parcelas permanentes de muestreo (Dauber et al. 2005, Licona et al. 2007). Por ejemplo, en La Chonta, Guarayos, Dauber et al. (2005) reportan tasas de crecimiento anual de 0,54 y 0,63 cm año-1 para Cedrela sp. y C. microchaete, respectivamente, cuyos valores fueron similares a nuestros resultados presentados en los cuadros 2 y 3 . Esta consistencia de valores entre ambas técnicas, también se observó en C. microchaete, P. ulei y A. colubrina del distrito Chiquitano, cuyos incrementos anuales, son similares a los obtenidos por Dauber et al. (2005) en sitios del mismo distrito. Para Ficus boliviana y Hymenaea courbaril en la región de estudio y sus proximidades, no existe información sobre tasas de crecimiento diamétrico. Sin embargo para A. cearensis en la Amazonía boliviana mediante métodos dendrocronológicos, Brienen y Zuidema (2006b), obtuvo datos similares a los obtenidos en este estudio. Por medio del análisis de los anillos de crecimiento en el norte Argentino para esta misma especie, se reportaron valores similares a los presentados en este estudio (Calzón y Palma 2008). Si bien la medición de árboles, en parcelas permanentes de muestreo, brinda datos confiables después de muchos años, incluso décadas de monitoreo, la información provista por esta metodología está limitada solo a una proporción muy reducida del bosque (Brienen y Zuidema 2006b). En general, las especies en estudio mostraron tasa de crecimiento más bajas que las esperadas, con excepción de F. boliviana (cuadros 2 y 3).

El periodo de medición en estas especies deberá extenderse por periodos más largos si se pretende determinar el diámetro alcanzado en 25 años o la edad para alcanzar el DMC. En este sentido, los anillos de crecimiento permitieron entender la variación existente a lo largo de la vida de los árboles (figuras 1 y 2). A su vez, en regiones donde las tasas de crecimiento son bajas y la información es casi inexistente, este análisis puede ser una alternativa para conocer los ritmos pasados y presentes del crecimiento que pueden dar respuestas a muchas interrogantes en el manejo forestal boliviano.

El manejo forestal en Bolivia con ciclos de rotación entre cosechas de 25 años, pretende dar teóricamente un flujo continuo de maderas a largo plazo (MDSP 1998). Sin embargo, ni aun las tasas de crecimiento superior a $1 \mathrm{~cm}$ año ${ }^{-1}$ de Ficus boliviana en la región de Guarayos permitirán que los árboles de esta especie en 25 años alcancen $70 \mathrm{~cm}$, DMC establecidos para esta especie. Mediante una estimación rápida de acuerdo al incremento medio anual de Ficus boliviana (cuadro 2), está especie solo alcanzó $27 \mathrm{~cm}$ de diámetro en 25 años. En la región de Guarayos, existen otras especies con tasas mucho más bajas como H. courbaril (cuadro 2), en 25 años solo acumuló la mitad que Ficus boliviana. Si bien existen árboles remanentes que pueden ser aprovechados en los próximos ciclos, la reducción en las tasas de crecimiento en diámetros más grandes (figuras 1 y 2), puede limitar a que solo una reducida cantidad de ejemplares con diámetros entre $40-50 \mathrm{~cm}$ alcancen los DMC en los próximos 25 años. Estos datos 
indican que los volúmenes de madera obtenidos en próximas cosechas serán menores (Brienen y Zuidema 2006b). Esta situación es mucho más crítica en el distrito Chiquitano. Allí, las especies con las tasas más altas no superan los 0,6 cm año-1 (cuadros 2 y 3), por lo que el crecimiento acumulado en 25 años no podría ser superior a los $14 \mathrm{~cm}$ de diámetro. En este distrito existen especies que poseen tasas de crecimiento mucho más bajas a las reportadas por Dauber et al (2005) y en este estudio (cuadros 2 y 3). Solo algunas especien lograrán superar los $10 \mathrm{~cm}$ de incremento diamétrico en 25 años (López 2010). Este escenario hace que los tiempos fijados para alcanzar un determinado diámetro sean altamente inconstantes entre especies y demasiado cortos. Por lo tanto, los ciclos de corte de 25 años claramente deberían extenderse a más de 50 años para bosques nativos tropicales del Cerrado boliviano (Brienen y Zuidema 2006b).

Finalmente, las pautas forestales deberán considerar otros aspectos relevantes para una buena gestión forestal sostenible, entre los cuales se encuentra la marcada diferencia del crecimiento diamétrico entre especies, y la variación del crecimiento de una misma especie en sitios y distritos con características ambientales diferentes. Por lo que imponer pautas fijas dentro de regiones distintas y para todas las especies, sin tener en cuenta las diferentes tasas de crecimiento, pondrá en riesgo considerables extensiones de bosques del Cerrado boliviano.

\section{CONCLUSIONES}

Este estudio ha demostrado que el análisis de los anillos de crecimiento aplicado a siete especies forestales del Cerrado boliviano, es una herramienta útil para obtener información ecológica aplicable al manejo forestal de los bosques tropicales secos. En comparación a las técnicas tradicionales que requieren años, incluso décadas de monitoreo para que los primeros resultados tengan validez, este método brinda tasas de crecimiento altamente consistentes con aquellas obtenidas por más de 10 años de medición en parcelas permanentes de muestreo. Los anillos de crecimiento indican que las siete especies forestales poseen tasas de crecimiento más lentas que las asumidas y sumamente variables entre especies en un mismo sitio. A su vez, hemos visto que una misma especie alcanza tasas de crecimiento muy diferentes entre sitios diferentes de un mismo distrito. Estos resultados tienen importantes implicaciones para el manejo forestal, por lo que imponer pautas fijas para todas las especies y regiones, sin considerar estas variaciones, sería equivocado y no conduciría en el largo plazo al manejo forestal sostenible de los bosques tropicales del Cerrado boliviano.

\section{AGRADECIMIENTOS}

Los autores agradecen el apoyo económico brindado por Education for Nature Program, World Wildlife Fund
(WWF), al Concejo Nacional de Ciencia y Tecnología (CONICET) de Argentina y al proyecto CRN2047 del Instituto Interamericano para la Investigación del Cambio Global (IAI). Por facilitar las tareas de campo agradecemos al Instituto Boliviano de Investigación Forestal (IBIF), a INPA Parquet, a la empresa Agroindustrial La Chonta y a las comunidades originarias de Santa Mónica, Santa Anita, Zapocó y Makanaté. Finalmente, agradecemos al Dr. José Reque por la lectura crítica del manuscrito.

\section{REFERENCIAS}

BOLFOR II (Proyecto de Manejo Forestal Sostenible, BO). 2008. Informe final. Santa Cruz, Bolivia. Proyecto, BOLFOR II. 179 p.

BOLFOR II (Proyecto de Manejo Forestal Sostenible, BO). 2009. Dossier Forestal. Santa Cruz, Bolivia. Proyecto BOLFOR II. 286 p.

Brienen RJW, PA Zuidema. 2005. Relating tree growth to rainfall in Bolivian rain forests: a test for six species using tree ring analysis. Oecologia 146: 1-12.

Brienen RJW, PA Zuidema. 2006a. Lifetime growth patterns and ages of Bolivian rain forest trees obtained by tree ring analysis. Ecology 94: 481-493.

Brienen RJW, PA Zuidema. 2006b. The use of tree rings in tropical forest management: Projecting timber yields of four Bolivian tree species. Forest Ecology and Management 226: 256-267.

Cabrera AL, A Willnk. 1973. Biogeografía de América Latina. OEA (Organización de los Estados Americanos). Washington, DC. 120 p.

Calzón ME, RM Palma. 2008. El roble criollo. XXIII Jornadas Forestales de Entre Ríos. Concordia, Argentina: 2-9

Cook ER, K Peters. 1981. The smoothing spline: A new approach to standardizing forest interior tree-ring width series for dendroclimatic studies. Tree-Ring Bulletin 41: 45-53.

Dauber E, TS Fredericksen, M Peña. 2005. Sustainability of timber harvesting in Bolivian tropical forests. Forest Ecology and Management 214: 294-304.

Di Rienzo J, M Balzarini, F Casanoves, L Gonzalez, E Tablada, C Robledo. 2009. Versión 2009. Grupo InfoStat, FCA, Universidad Nacional de Córdoba, Argentina.

Holmes RL. 1983. Computer-assisted quality control in tree-ring dating and measurement. Tree-Ring Bulletin 43: 69-78.

ITTO (International Tropical Timber Organization, JP). 1996. Manual de Identificación de Especies Forestales de la Subregión Andina, IANIA-OIMT. Lima, Perú. Grupo Stella. $489 \mathrm{p}$.

Kant S. 2004. Economics of sustainable forest management. Forest Policy and Economics 6: 197-203.

Killeen JT, E Garcia, GS Berck. 1993. Guía de árboles de Bolivia. Herbario Nacional de Bolivia, Missouri Botanical Garden. La Paz, Bolivia. Quipus S.R.L. 958 p.

Licona JC, M Peña-Claros, B Mostacedo. 2007. Composición Florística, Estructura y Dinámica de un Bosque Amazónico aprovechado a diferentes intensidades en Pando, Bolivia. Santa Cruz, Bolivia. Proyecto BOLFOR / Instituto Boliviano de Investigación Forestal. 60 p.

López L. 2003. Estudio de anillos de crecimiento en once especies forestales de Santa Cruz-Bolivia. Tesis Ingeniero Fo- 
restal. Universidad Autónoma Gabriel René Moreno, Santa Cruz, Bolivia. 147 p.

López L. 2010. Una aproximación dendrocronológica a la ecología y el manejo de los bosques tropicales secos del Cerrado Boliviano. Tesis doctoral. San Carlos de Bariloche, Argentina. Universidad Nacional del Comahue. 225 p.

López L, R Villalba. 2010. Climate Influences on the Radial Growth of Centrolobium microchaete, a Valuable Timber Species from the Tropical Dry Forests in Bolivia. Biotropica 43: 41-49.

MDSMA (Ministerio de Desarrollo Sostenible y Medio Ambiente, BO). 1997. Nueva Ley Forestal. Ley $N^{\circ} 1700$ del 12 de julio de 1996. La Paz, Bolivia. Ministerio de Desarrollo Sostenible y Medio ambiente.

MDSP (Ministerio de Desarrollo Sostenible y Planificación, BO). 1998. Normas técnicas para la elaboración de instrumentos de manejo forestal (inventarios, planes de manejo, planes operativos, mapas) en propiedades privadas o concesiones con superficies mayores a 200 hectáreas. Resolución Ministerial N²48/98. La Paz, Bolivia. Ministerio de Desarrollo Sostenible y Planificación.

Mostacedo B, Z Villegas, JC Licona, A Alarcón, D Villarroel, M Peña-Claros, TS Fredericksen. 2009. Ecología y Silvicultura de los Principales Bosques Tropicales de Bolivia. Santa Cruz, Bolivia. Instituto Boliviano de Investigación Forestal. 142 p.

Navarro G, M Maldonado. 2004. Geografía ecológica de Bolivia: Vegetación y Ambientes Acuáticos. Santa Cruz, Bolivia. Centro de Ecología Simón Patiño. 719 p.

Schöngart J. 2008. Growth-Oriented Logging (GOL): A new concept towards sustainable forest management in Central Amazonian várzea floodplains. Forest Ecology and Management 256: 46-58.

Schulman E. 1956. Dendroclimatic Changes in Semiarid America. Tucson, USA. University of Arizona Press. 142 p.

Stokes MA, TL Smiley. 1968. An introduction to tree-ring dating. Chicago, USA. University of Chicago Press. 73 p.

Recibido: 07.02.12

Aceptado: 11.06.12 
